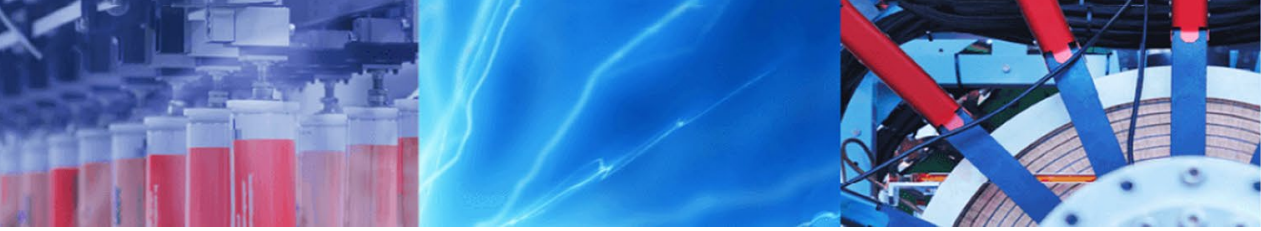

Research Article

\title{
Influence of chemical modification of kenaf fiber on XGNP-PP nano-biocomposites
}

\author{
Christopher Igwe Idumah ${ }^{1}$. James E. Ogbu ${ }^{2} \cdot$ J. U. Ndem ${ }^{2} \cdot$ Viola Obiana $^{2}$
}

(c) Springer Nature Switzerland AG 2019

\begin{abstract}
Very recently emerging trends in polymer nanotechnology has revealed tremendous upsurge in application of graphene and derivatives in development of multifunctional nanocomposites. In present work, recycled polypropylene filled with increasing concentration of kenaf flour modified with sodium hydroxide/cetyl-trimethylammonium bromide (C-TAB) and filled with 3-phr graphene nanoplatelets (xGNP) hybrid nanocomposites were compatibilized using maleic anhydride grafted polypropylene and prepared via melt-intercalation using co-rotating twin-screw extruder subsequented by injection molding. The mechanical and morphological properties of $\mathrm{NaOH}-\mathrm{C}-\mathrm{TAB}$ modified materials (T-KRPPG) were studied and compared with untreated (U-KRPPG) samples. Transmission electron microscopy, field emission scanning electron microscope, Fourier transform infra-red spectroscopy (FTIR) and X-ray diffraction (XRD) analysis were applied in characterizing the microstructure and morphology of the materials. Mechanical tests revealed that tensile strength of T-KRPPG nanocomposites were superior in comparison with untreated (U-KRPPG) samples. In addition, the flexural strength, flexural modulus, and Young's modulus of C-TAB treated kenaf XGNP (T-KRPPG) nanocomposites were superior in comparison with the untreated (U-KRPPG) samples. The morphological images of the tensile fractured surface of modified T-KRPPG nanocomposites revealed reduction of microholes and fibres aggregation signifying enhanced xGNPfiber-matrix inter-facial adhesion due to kenaf flour modification with $\mathrm{NaOH} / \mathrm{C}-\mathrm{TAB}$. XRD analysis revealed exfoliation and homogeneous separation of XGNP in matrix. FTIR analysis revealed no variations in peak positions of XGNP to suggest any significant chemical interactions between XGNP and other componenets.
\end{abstract}

Keywords Cetyl-trimethylammonium bromide Exfoliated graphene nanoplatelets $\cdot$ Thermal properties $\cdot$ Mechanical properties

\section{Introduction}

The versatility of plastic based materials in emerging technologies has been broadened by nanotechnology. The increasing use of plastic materials has added to escalating global solid waste issues with plastic materials accounting significantly for the upsurge and mostly comprising of polyolefins such as polypropylene and polyethylene. Notably in developing economies, open-burning, unregulated dumping, and landfilling are main routes of disposal of these wastes. Hence, eco-benign and energetically low cost route of utilizing this huge quantity of waste has become a global issue. Thus, policy makers around the globe having come to realization that unchecked dumping and open-burning are not eco-benign routes of disposing these waste has called for discontinuation while advocating for recycling and reusing of these polymer based solidwastes as panacea to this challenges [1]. Due to increasing environmental and ecological awareness, thermoplastic based composites filled with low-cost lignocellulose fibers have aroused great interests because of accruable benefits such as reduction in wearing of processing equipments

Christopher Igwe Idumah, idugoldengate@yahoo.com | 'Enhanced Polymer Research Group, Department of Polymer Engineering, Faculty of Chemical Engineering, Universiti Teknologi Malaysia, 81310 Skudai, Johor, Malaysia. ${ }^{2}$ Ebonyi State University, Abakaliki, Nigeria.

SN Applied Sciences (2019) 1:1261 | https://doi.org/10.1007/s42452-019-1319-1 
and low temperatures of processing when compared with the commonly available mineral fillers thereby playing a significant role in saving energy during fabrication of composites [2].

Additionally, composites fabricated through the inclusion of renewable natural fibers such as wood, corn-cob, rice, and abaca flours in polymers are durable, eco-benign, and biodegradable. However, use of lignocellulose fibers as reinforcing materials have been limited due to inherent propensity to thermally degrade at elevated processing temperatures and affinity to absorb moisture from the environment which distorts material dimensional stability, mechanical and flame retardant properties while reducing durability of the end product. Also, Van der Waals forces, formation of chemical bonds, and mechanical inter-locking between matrix and fiber affect inter-facial strength of composites. Hence, attainment of good mechanical properties for engineering composites has been a drawback due to the high hydrophilic affinity of natural fibers caused by the presence of hydroxyl groups in cellulose and apolar nature of thermoplastics which hinder effective interaction between matrix and fiber. In addition, the absorption of moisture from the environment into crevices and microgaps of composites potentially result in swelling, fungal proliferation, formation of micro-cracks, and deterioration of the mechanical properties of these materials.

Thus, in order to provide a panacea to these challenges, chemical modification of fiber surface structure and appropriate use of compatibilizing agents composed of both hydrophilic and hydrophobic components, with potentials of providing chemical bonding of fiber and matrix, while significantly reducing the inherent hydrophilic affinity of fiber and also decreasing the polarity gap between matrix and fibre with improvements in inter-facial adhesion have been proffered. In addition, formulated inclusion of nanoparticles has propagated improvement in most properties of composites.

The discovery of graphene and emerging trends in graphene based polymer nanocomposites are important land-marks in the field of nano-science [3]. These carbon nanomaterials have several grades and sizes with thickness and width ranging from " $1-20 \mathrm{~nm}$ " and "1-50 microns respectively" [4]. Thus, recently the scope of nano-engineering has expanded with industrial application of nanotechnology extended to areas such as energy storage, computing, biomedical, drug delivery and so on [5]. Graphene is a hexagonally packed lattice single layer $s p^{2}$ bonded carbon atoms, having a structure exhibiting interesting properties such as excellent thermal conductivity $\left(3000-5000 \mathrm{Wm}^{-1} \mathrm{~K}^{-1}\right)$ and optical transparency ( 97.3\%), high young modulus ( 1 TPa), and high transport mobility at $25^{\circ} \mathrm{C}\left(\sim 10,000 \mathrm{~cm}^{2} \mathrm{v}^{-1} \mathrm{~S}^{-1}\right)$. Exfoliated graphene nanoplatelets ( $x$ GNP) are ultra-thin short stacks of graphene sheets composed of graphite nanoparticles with multi-functional properties [6]. The platelet morphology of XGNP and its nano-size enhances its effectiveness at providing very good properties, while its inherent pure graphitic composition enhances its thermal and electrical conductivity. Other interesting properties of XGNP include wide surface area, high aspect ratio, superior mechanical and flame retardant properties $[5,6]$. However, emerging researches have revealed that low inclusion of $x G N P$, other carbon derivatives and nanofillers into plastics has resulted in fabrication of polymer nanocomposites with superior mechanical, thermal and flame retardant properties [7].

Presently, this research investigates the influence of cetyl-trimethylammonium bromide modified kenaf flour on recycled polypropylene (RPP) graphene nanoplatelets (3 phr) nanocomposites at varying compositions of fibre. Here, a dual process technique of fibre surface modification was applied to influence the hydrophilic affinity of kenaf flour inorder to reduce polarity difference existing between matrix and fibre which is anticipated to enhance their interfacial adhesion. Thus, cetyl-trimethylammonium bromide (C-TAB) acted as a modifier and cationicsurfactant capable of facilitating chemical interaction with $\mathrm{NaOH}$ treated kenaf flour through enhancement of the electrostatic, interfacial interaction, and surface absorbtion of the fiber. The inclusion of 3-phr graphene nanoplatelets is also expected to improve mechanical properties of the nanocomposites. Accruable benefits of this technique include the short duration of fibre modification and eco-benign attributes.

\section{Experimental}

\subsection{Materials and methods}

Materials utilized for this investigation included exfoliated graphene nanoplatelets (xGNP), GNP-M5 grades (99.5\% carbon) of mean diameter and thickness of $5 \mathrm{~mm}$ and $6 \mathrm{~nm}$ respectively, purchased as dry flour from XG Sciences Incorporated East Lansing, MI, USA, and applied as received. Results of laboratory BET surface measurements revealed samples surface area to be $158 \mathrm{~m}^{2} / \mathrm{g}$. The concentrations of XGNP were kept constant at 3 parts per hundred (phr) of total composites. Recycled polypropylene (RPP) was obtained from waste polypropylene-based shopping-bags. These materials were cleaned, cut into bits, and subsequently dried in a vacuum oven at $80^{\circ} \mathrm{C}$ for $24 \mathrm{~h}$. After washing off extraneous dirts, the materials were finally converted into tiny beads. Kenaf flour which was obtained from Malaysian Agricultural and Development Institute (MARDI), Kuala Lumpur was ground and 
sieved using a sieve-shaker equipment having a mesh of $<500 \mu \mathrm{m}$ to obtain kenaf flour of sizes $<500 \mu \mathrm{m}$ in longitudinal direction and was subsequently dried for $24 \mathrm{~h}$ in an oven maintained at $80^{\circ} \mathrm{C}$. Cetyl-trimethylammonium bromide (Merck) and sodium hydroxide (Merck) were used in treating kenaf flour. Initially, dried kenaf flour was treated using $5 \% \mathrm{NaOH}$ (aq) and further modified for about $10 \mathrm{~min}$ using $0.5 \%$ C-TAB (aq). This was subsequently followed by washing using distilled water and open-air drying. The compatibilizer MA-g-PP which was purchased from DuPont, Dow Elastomers, and Wilmington DE, USA was dried at temperature of $60^{\circ} \mathrm{C}$ for $5 \mathrm{~h}$. Kenaf flour processed as elucidated above was initially mixed completely with RPP beads at 20/80, 25/75, 30/70, and 35/65 wt\% mixing ratios and designated as samples 2, 3, 4 and 5 respectively.

The composites were melt-extruded utilizing Brabender-PL-2000-Plastic-Coder counter-rotating twin-screw extruder (with a rod-die of $4 \mathrm{~mm}, \mathrm{~L} / \mathrm{D}=30$ and $\mathrm{D}=25 \mathrm{~mm}$ ). Extruder melt-pressure was about 12 bars with optimized temperature maintained at $185^{\circ} \mathrm{C}$ from feed to die-head zone. All materials were melt-intercalated in a single-step process with increasing concentrations of kenaf flour according to the sample-formulation. The screw-speed was permanently maintained at $60 \mathrm{rpm}$. Subsequently, the extrudate was immediately pelletized after melt intercalation. Prior to injection-molding to standard-samples, the pellets were dried in oven at $80^{\circ} \mathrm{C}$ for $24 \mathrm{~h}$ so as to eliminate moisture. JSW-Muraron-Japan injection molding-machine of model-NIOOB-11 was utilized in preparing the flexural, tensile, and notched-lzod impact-tests-specimens at a temperature range of $185-200^{\circ} \mathrm{C}$. The calculated Brunauer, Emmett and Teller (BET) specific surface area of GNP powder utilized in present study is $158 \mathrm{~m}^{2} / \mathrm{g}$ obtained from laboratory evaluation as elucidated below.

\subsection{Determination of BET specific surface area}

The Micrometrics-Gemini-V surface-area analyzer, with isotherm-nitrogen adsorption, positioned at $77 \mathrm{~K}$, was applied in determining the Brunauer, Emmett and Teller (BET) specific surface area of GNP flour. Prior BET analysis, samples underwent degassing at atmospheric pressure for $4 \mathrm{~h}$ at $623 \mathrm{~K}$. Equation (1) was utilized to calculate the BET specific surface area [8]. The parameters for Eq. (1) are expressed in Table 1.

Table 1 Elucidation of BET equation parameters
$\mathrm{S}=\mathrm{V}_{\mathrm{m}} \mathrm{Na} / 22,400$

\section{Characterizations}

\subsection{Morphology analysis}

Field emission scanning electron microscopy (FESEM) was utilized in analyzing the distribution of XGNP and kenaf flour in matrix via a Hitachi-S-4800 equipment. A Balzers-UnionMED-010 coater was used in coating the impact fractured surfaces of untreated UK-RPPG and treated TK-RPPG nanocomposites and micrographs depicting the fractured surfaces of the materials were pictured accordingly. TEM images were derived utilizing Zeiss Libra-120 TEM equipment via samples of dimensions $0.2 \mathrm{~cm} \times 1 \mathrm{~cm}$.

\subsection{Mechanical properties}

In accordance with ASTM D 638 standard, tensile samples were tested utilizing the universal materials EZ-20KN-LLYORD tensile testing equipment set at a cross-head speed of $50 \mathrm{~mm} / \mathrm{min}$. Five samples were tested from each composition in addition with untreated samples for comparative analysis. The three-point centre loading technique was used for flexural testing according to ASTM D790. Samples for flexural tests were placed on a supported beam with span-distance of $50 \mathrm{~mm}$ and strain-rate (compressed-speed) of $3 \mathrm{~mm} / \mathrm{min}$ [9]. Notched-impact-test in accordance with ASTM D 256 was conducted at room temperature using IzodToyoseiki-impact testing equipment (11 J).

\subsection{X-ray diffraction analysis}

X'Pert-X-ray-diffractometer (Siemens-XRD-D5000) and $\mathrm{Ni}$-filtered-Cu-Ka radiation at an angular-incidence of $10^{\circ}-80^{\circ}$ ( $2 \Theta$ angle-range) was applied in obtaining X-ray diffraction-patterns. XRD scanning of XGNP flour and composites were conducted using a time of exposure of $120 \mathrm{~s}$ at $40 \mathrm{kV}$ and $50 \mathrm{~mA}$. Bragg's Eq. (2) was used in deriving the diffraction patterns used in evaluating degree of XGNP and T-KF distribution in matrix.

$\mathrm{n} \lambda=2 \mathrm{~d} \sin \Theta$
$V_{m}=$ Calculated value from $1 /($ slope + intercept $)$ in graph of $1 /\left(V_{a}\left(P_{o} / P\right)-1\right)$ versus $P / P_{o}$
$N=$ Avogadro constant given as $6.0 \times 10^{23} \mathrm{~mol}^{-1}$
$a=$ Cross sectional area of single adsorbate molecule in meters square $\left(0.195 \mathrm{~nm}^{2}\right.$ for krypton and
$0.162 \mathrm{~nm}^{2}$ for nitrogen)
$\mathrm{m}=$ Mass of GNP test flour in grams
22,400 Volume of $1 \mathrm{~mol}$ of adsorbate gas at STP allowing for slight shift from the ideal, in milliliters

SN Applied Sciences 
where $\Theta=$ angle-in-between incident-rays and crystalsurface, $\lambda=$ wave-length of X-ray $=0.154 \mathrm{~nm}(\mathrm{Cu} \mathrm{Ka}), \mathrm{n}=1$ and $d=$ spacing-between-atom-layers.

\subsection{Fourier transform infrared spectroscopy}

Fourier transform infrared spectroscopy (FTIR) of samples was carried out via Perkin-Elmer-1600 infrared spectroscopy ( $\mathrm{KBr}$ technique) using a ratio of 1:100 prior to conversion of samples to thin pellets. A Nicolet-AVATAR-360 at 32 scans with a resolution of $4 \mathrm{~cm}^{-1}$ and within the wave number range of $4000 \mathrm{~cm}^{-1}-1000 \mathrm{~cm}^{-1}$ was utilized in obtaining FTIR spectra of coated-pellets. Significant points of transmittance-peaks were determined using the "findpeak-tool" provided by Nicolet-OMNIC-5.01 software.

\section{Results and discussion}

\subsection{Morphological analysis}

\subsubsection{Field emission scanning electron microscopy (FESEM)}

Figure 1 shows the FESEM images of the fractured surface of tensile specimens of (a) untreated kenaf flour (U-KRPPG) and (b) C-TAB treated kenaf flour (T-KRPPG) nanocomposites. The micrograph of the untreated U-KRPPG nanocomposites in Fig. 1a revealed existence of fiber-matrix interfacial holes, spaces and voids implying that the interfacial adhesion existing between XGNP, RPP and kenaf flour is weak. Moreover, due to high fiber-fiber interactions, agglomeration of fibres results in formation of cracks and voids as depicted by arrows in Fig. 1a. However, for C-TAB treated T-KRPP composites, the interfacial voids existing between $x G N P$, fiber and matrix is significantly reduced as revealed in Fig. $1 \mathrm{~b}$. As a result of the property variation in kenaf flour due to C-TAB treatment in addition to the adsorption of molecules of C-TAB on surface of kenaf flour, the gap in polarity existing between the fiber and the matrix decreased thereby resulting in the enhancement of effective distribution of XGNP and kenaf flour in RPP matrix thereby reducing the microvoids within the matrix and fiber. This is evidenced by enhanced mechanical properties of T-KRPPG nanocomposites.

A homogenous distribution of nanoplatelets in composites due to inclusion of $3 \mathrm{phr}$ of XGNP devoid of agglomeration signs or over-stacking was observed in the materials as revealed in Fig. 2a-c. The graphene sheets were intact and seen spearing out of the blend revealing effective distribution in matrix. The uniform distribution of $x G N P$ in matrix is thought to enhance mechanical properties of the materials. Hans et al. opined that XGNP and kenaf flour interactions were physical since XGNP physically absorbed on the surface of the fibre with nil chemical interaction occurring between them [1].

\subsubsection{Transmission electron microscopy (TEM)}

The extent of distribution of nanofillers and microfiber fillers in matrix relates to the degree of properties enhancement in nanocomposite materials. Inorder to attain better insight into the degree of distribution of
Fig. 1 a FESEM micrograph of Tensile-fractured surface of U-KRPPG and $\mathbf{b}$ T-KRPPG nanocomposites

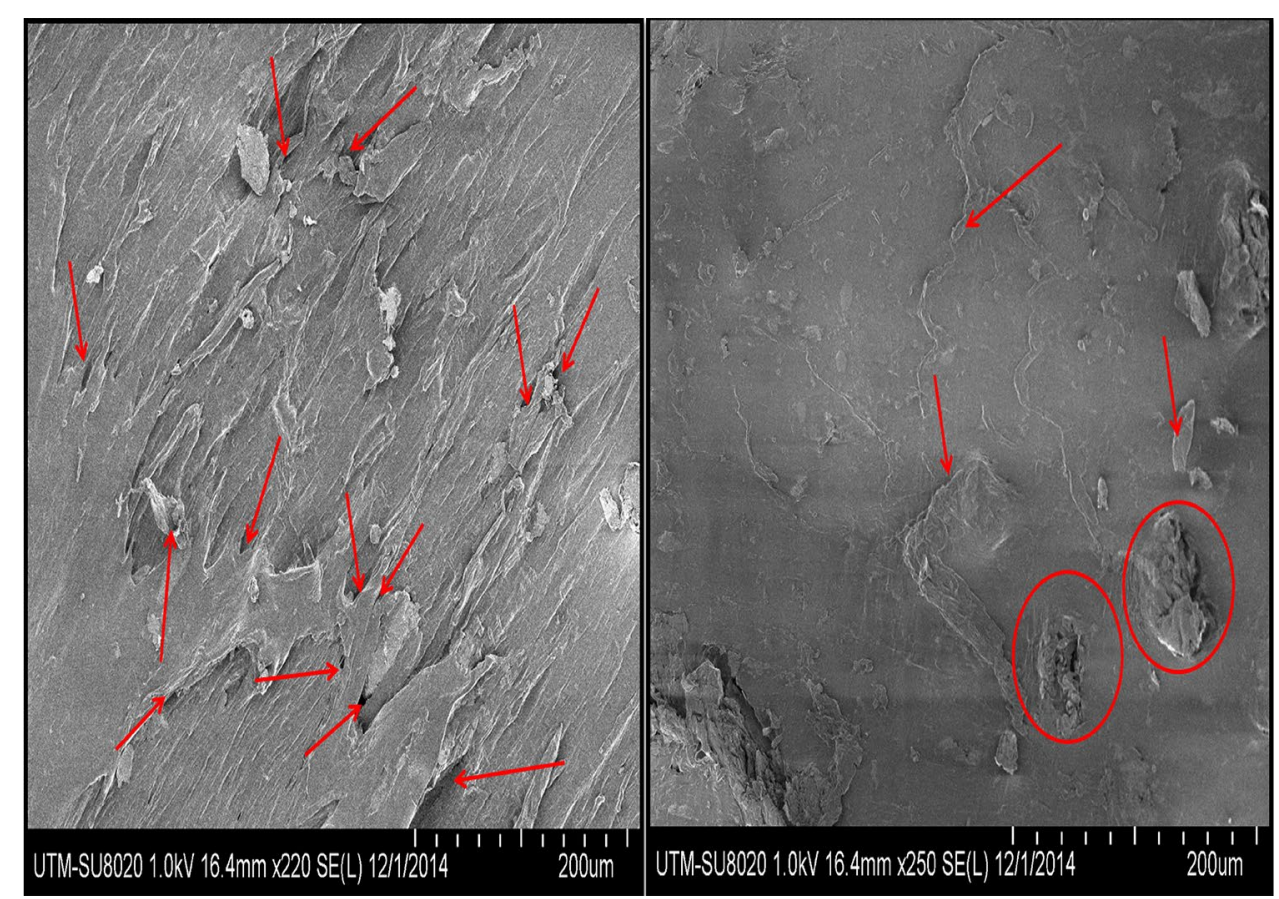



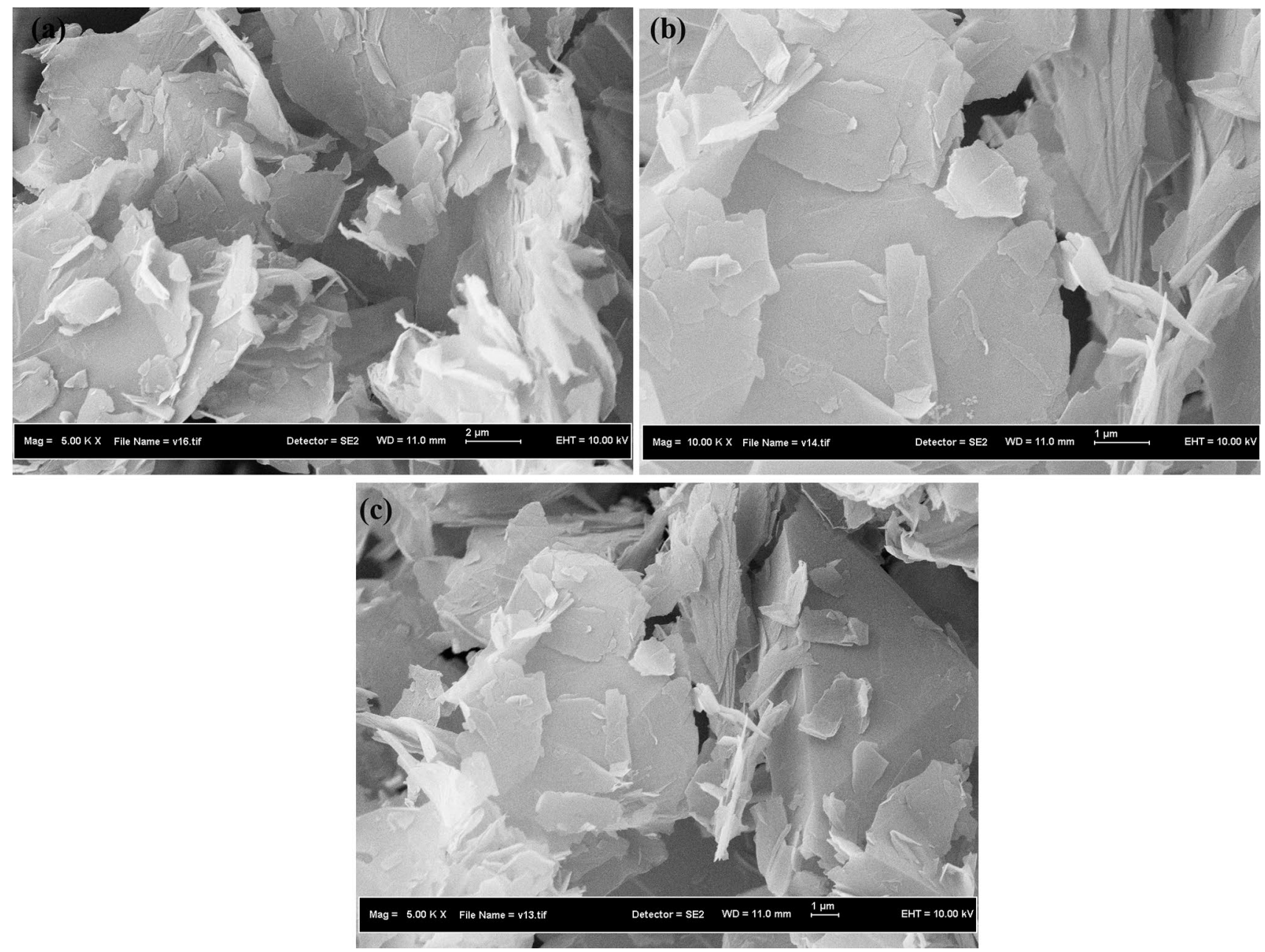

Fig. 2 FESEM microgragh of instances of xGNP exfoliated state in the nanocomposites

$x$ GNP and kenaf microparticles in matrix, TEM images were obtained from thin sections of specimens exhibiting dimensions of $0.2 \mathrm{~cm} \times 1 \mathrm{~cm}$. Figure $3 a$, b reveals the TEM images of thin-sections of untreated U-KRPPG and chemically modified T-KRPPG nanocomposites respectively. From Fig. 3a the presence of microcracks, holes and voids as depicted by the arrows reveals poor interfacial interaction between untreated kenaf, RPP and XGNP. However, for $\mathrm{NaOH}$ and C-TAB treated T-KRPP nanocomposites, the interfacial voids existing between $x G N P$, fiber and matrix is almost extinct as revealed in Fig. $3 b$. Due to changes in attributes of kenaf flour as a result of the chemical modification using $\mathrm{NaOH} / \mathrm{C}-\mathrm{TAB}$, the polarity-gap existing between the fiber and matrix diminished significantly thereby enhancing effective distribution of XGNP and kenaf flour in RPP matrix which subsequently reduced the microvoids within the matrix and fiber. This is evidenced by enhanced mechanical properties of T-KRPPG nanocomposites.

\subsection{Mechanical properties}

\subsubsection{Tensile properties}

The trend of tensile strength (TS) of both untreated (U-KRPPG) and treated kenaf flour reinforced $x G N P-R P P$ (T-KRPPG) nanocomposites at increasing content of fiber are shown in Fig. 4. Increasing concentrations of fibre resulted in a gradual decrease of TS. At a constant inclusion of 3-phr xGNP and lower inclusion of fibre, higher values of TS occur indicating enhanced interaction between fiber and matrix. An increasing content of kenaf flour escalates propensity of microvoids formation in the region of filler-matrix interface and agglomeration of fibre as result of escalating fiber-fiber interaction. Hence, TS decreases with increasing concentration of fiber in the nanocomposites. However, values of TS of chemically modified kenaf flour graphene reinforced RPP nanocomposites were observed to exhibit superiority to 
Fig. 3 a TEM micrograph of Tensile-fractured surface of U-KRPPG and $\mathbf{b}$ T-KRPPG nanocomposites

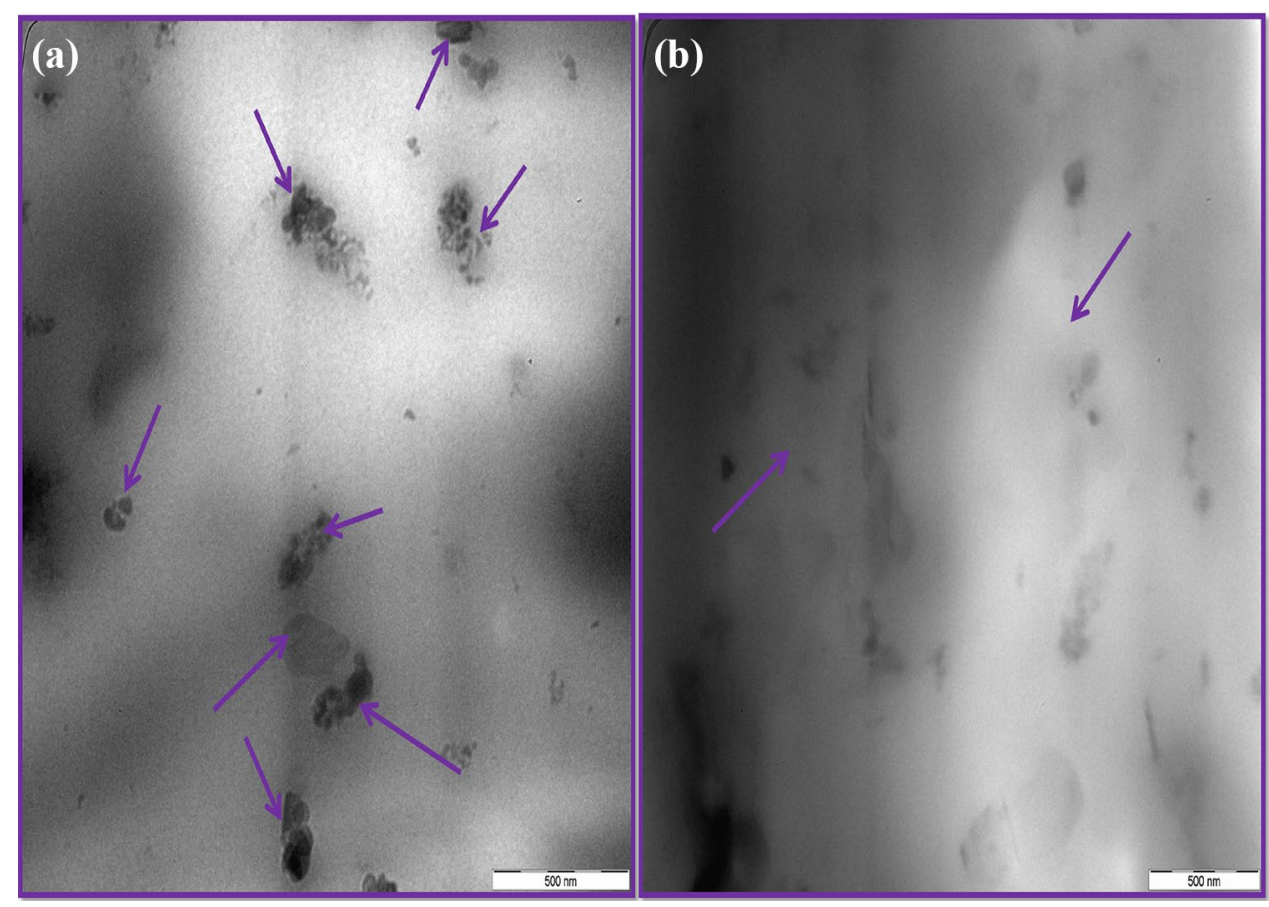

Fig. 4 Tensile strength of U-KRPPG and T-KRPPG nanocomposites

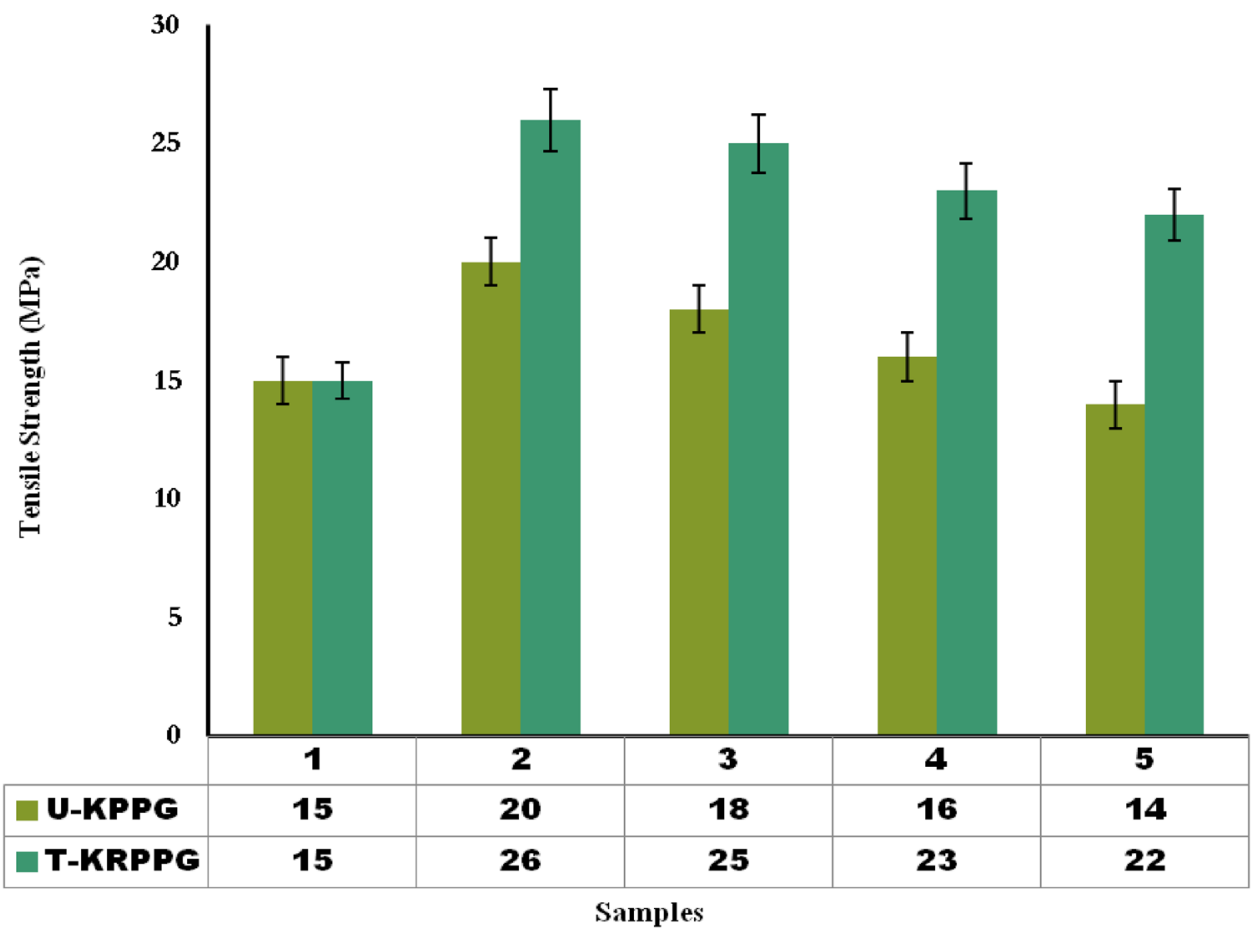

the values of the corresponding untreated samples at all ratios of mixing. This implies that chemically modifying kenaf flour using C-TAB enhanced interfacial-adhesion existing between the filler and matrix. Also, inclusion of stiff nanoparticles reduced tensile strength of nanocomposites [1]. Thus, improvement of tensile strength at low
fibre-xGNP inclusion occurred because tensile strength of composites is mainly determined by filler-fraction and the compatibility/or interfacial adhesion between the matrix, micro-natural-fiber fillers and nanoparticles [1, $3,5]$. 
Fig. 5 Young's modulus of U-KRPPG and K-RPPG nanocomposites

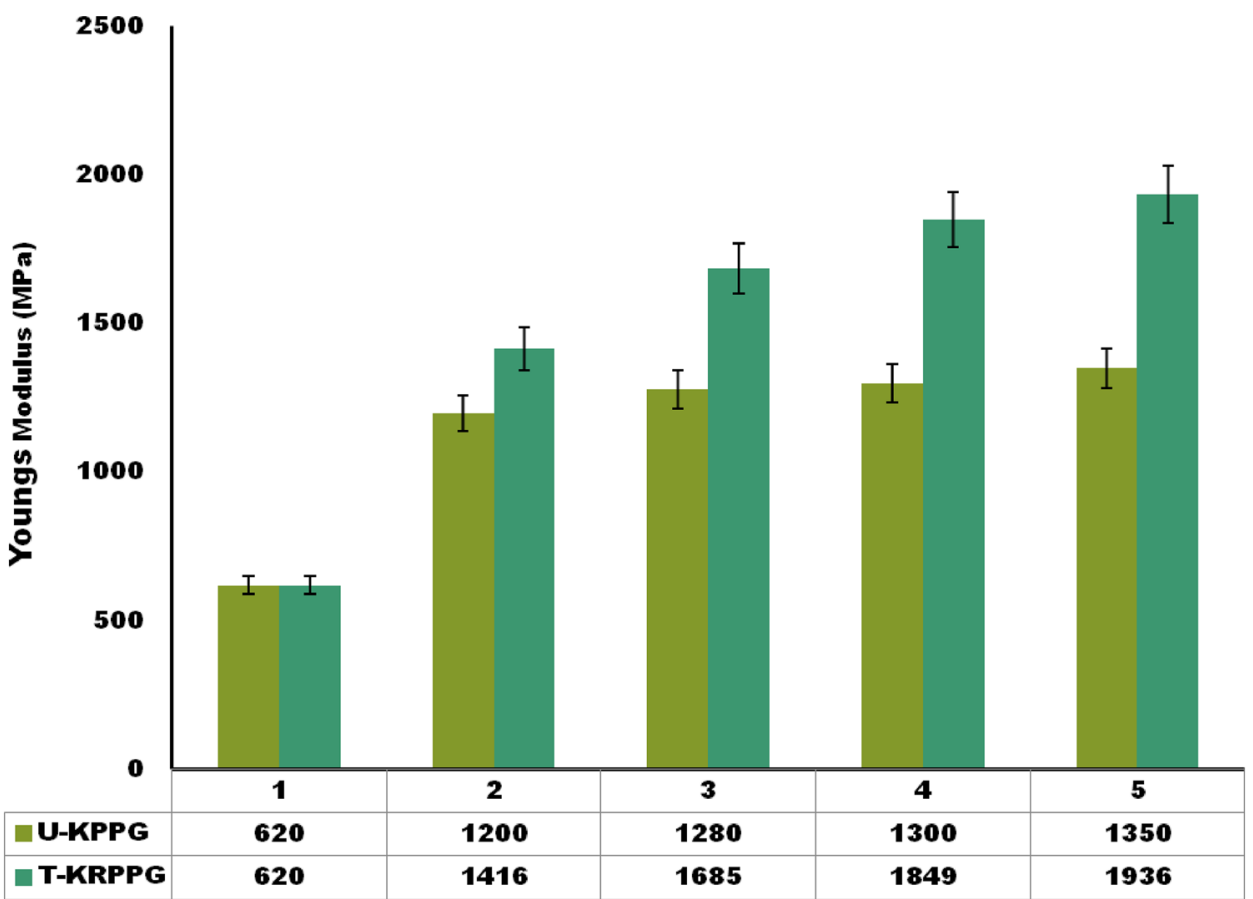

\subsubsection{Youngs modulus}

The trend of Young's modulus (YM) of the nanocomposites at varying fiber composition is shown in Fig. 5. The inclusion of 3-phr xGNP and varying fiber composition is anticipated to increase the composites YM because of the rigidity of the microfibres and nanoparticles filler in the soft RPP matrix. Evidently from Fig. 5, increases in both untreated and chemically modified kenaf flour content in the RPP matrix reinforced with graphene nanoplatelets lead to increasing values of YM values in the materials. However, chemically modified kenaf RPP nanocomposites exhibit superior modulus in comparison with the untreated samples. Studies have revealed that crystallites exhibits superior modulus when compared with amorphous substances and has potentials of raising the modulus input of matrix to the overall modulus of the composite [10]. The initial treatment of raw kenaf flour with $\mathrm{NaOH}$ in addition to its subsequent modification with C-TAB probably enabled the surface to attain a certain degree of crystallinity which is thought to dominate the entire bulk thereby resulting in enhancement of the YM of the chemically modified kenaf flour-RPPG nanocomposites. Moreover, inclusion of rigid microfiber and nanoparticles fillers into the matrix resulted in reduction of the mobility of the polymer and subsequent enhancement of values of YM in the materials.

\subsubsection{Flexural properties}

Figures 6 and 7 shows the trends of flexural modulus (FM) and flexural strength (FS) of untreated and chemically modified kenaf flour nanocomposites at varying fiber compositions respectively. It is evident from the graphs that inclusion of both $x G N P$, raw and chemically modified kenaf flour in the RPP matrix have significantly improved both FM and FS values of the materials. It is thought that the uniform and homogeneous dispersion of xGNP in matrix, in addition to effective mechanical inter-locking and entanglement of the matrix with kenaf flour would have lead to this improvement. Also, improved interfacial adhesion between matrix, micro-fiber, and nanofillers may be ascribed to effective compatibilization by maleic anhydride grafted polypropylene (MA-g-PP) which resulted in hindrance of matrix-deformation at the elasticzone and subsequent modulus enhancement. Additionally, the arrangement of XGNP in the flow direction during extrusion and injection molding may have enhanced the drastic improvement in material flexural modulus $[11,12]$. However, treated kenaf flour-xGNP-RPP nanocomposites exhibited superior $\mathrm{FM}$ and $\mathrm{FS}$ values in comparison with the untreated materials, revealing that $\mathrm{NaOH}$ and $\mathrm{C}-\mathrm{TAB}$ treatment of kenaf flour enhanced efficient transfer of stress from the polymer matrix to fiber. From Fig. 6, the values of FM for chemically modified kenaf flour xGNP-RPP 
Fig. 6 Flexural modulus of U-KRPPG and T-KRPPG nanocomposites
Fig. 7 Flexural strength of U-KRPPG and T-KRPPG nanocomposites
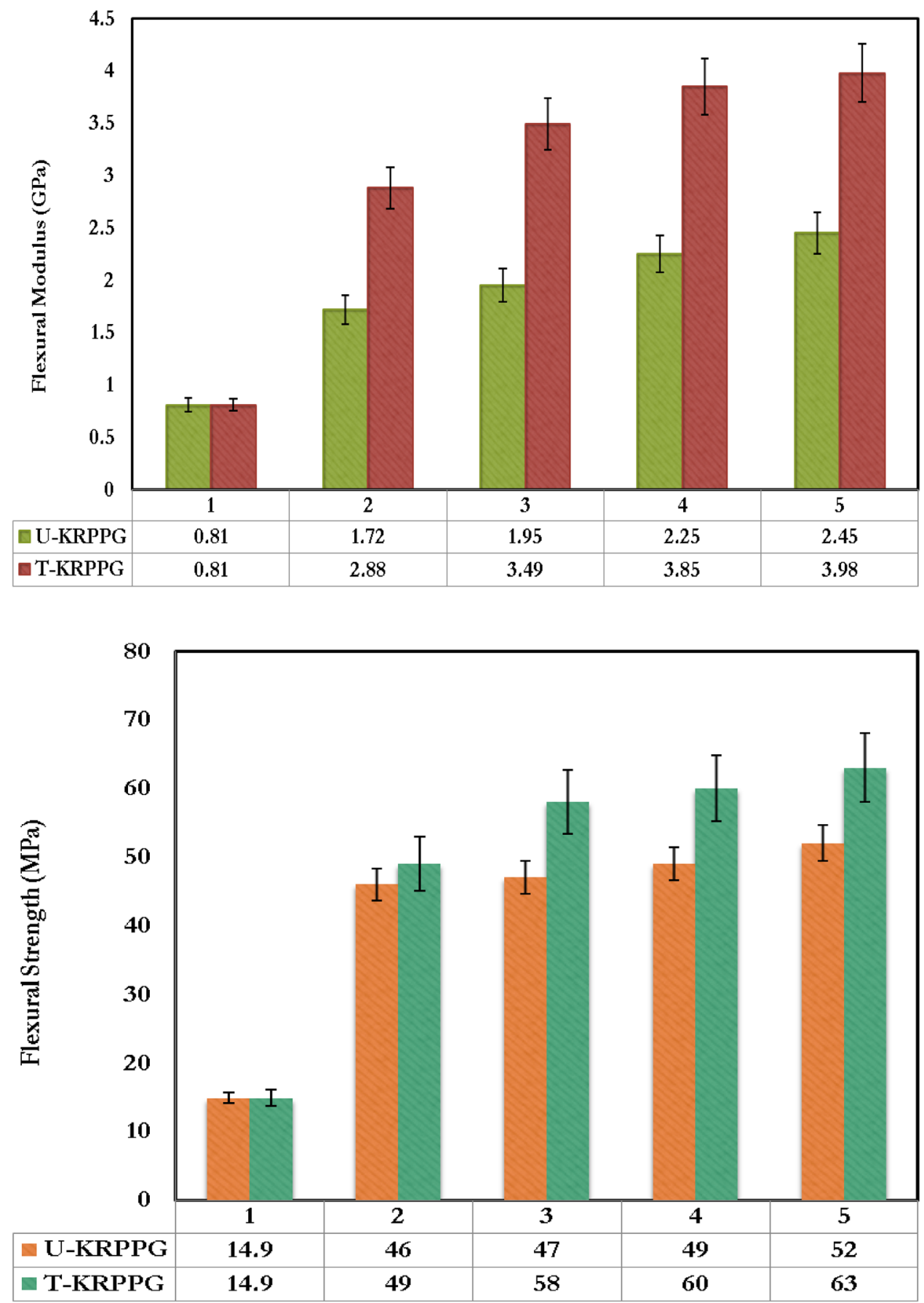

nanocomposites increased with increasing inclusion of kenaf flour. Inclusion of kenaf flour and stiff $x G N P$ restricted the rubbery modulus and mobility of RPP matrix because both fillers possesses high modulus. It is thought that RPP matrix effectively wetted all the filler particles at these ratios of mixing thereby enhancing the filler-matrix interfacial adhesion enough to propagate efficient transfer of stress from the matrix to the filler resulting in higher values of FM with increasing composition of kenaf flour in the materials. The effect of interfacial-bonding and fiber distribution in the matrix on flexural properties caused the stress applied to uniformly distribute in the chemically modified kenaf flour RPP nanocomposites thereby elevating their FM and FS values in comparison with the raw samples.

\subsubsection{Izod impact strength}

The essence of Izod impact strength testing is to discern materials resistance to impact fracture. The impact 
Fig. 8 Izod impact strength of U-KRPPG and T-KRPPG nanocomposites

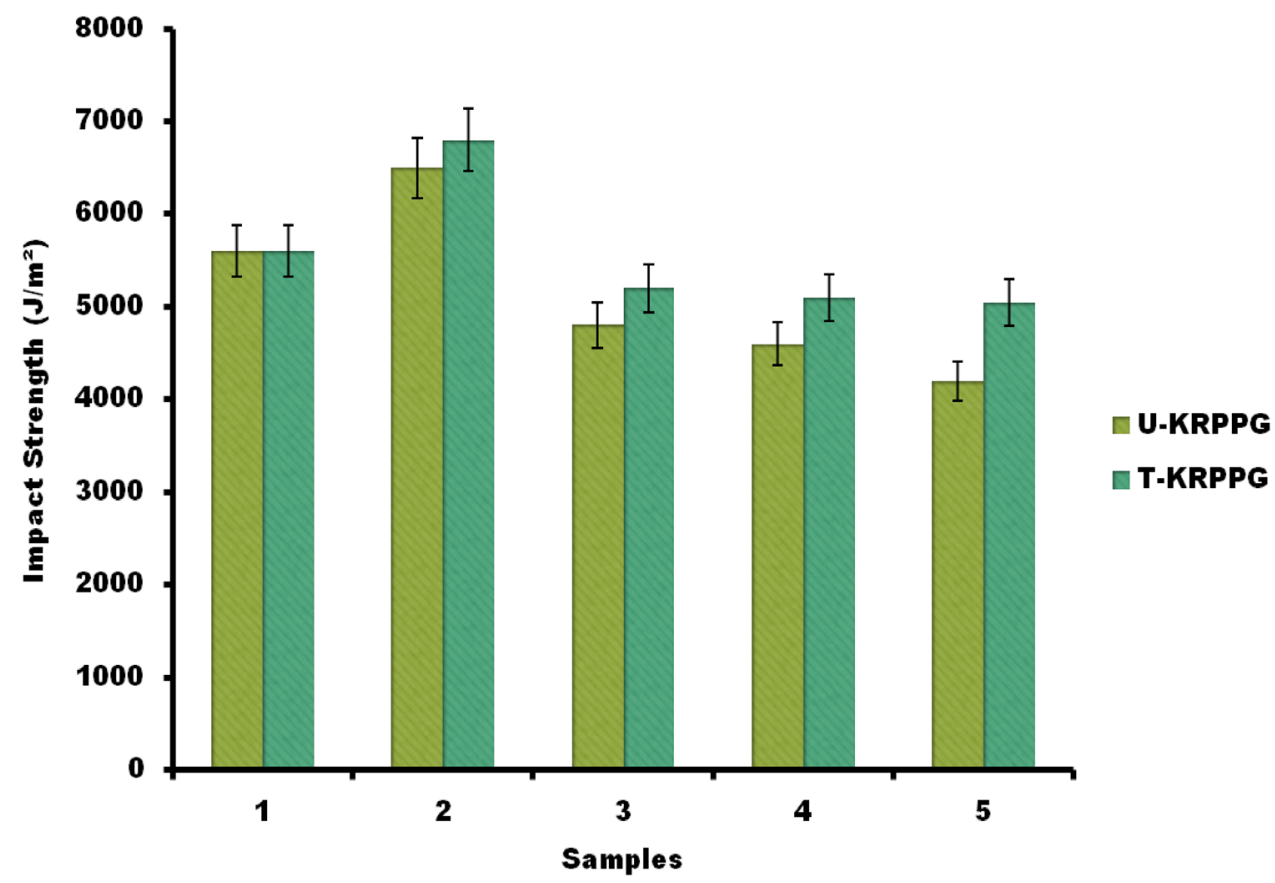

strength (IS) of a material gives insightful information as to the capability of the material to put up resistance to an instant impact. The notched Izod impact strength (IS) behavior of the materials at varying composition of kenaf flour and constant 3-phr xGNP content are shown in Fig. 8. The materials displayed a decreasing IS with increasing concentration of micro-fiber fillers. Higher values of IS exhibited by the materials at lower content of kenaf flour is ascribed to their propensity for higher energy absorbtion for initiation of crack. Moreover, $x$ GNP has the propensity of inducing nucleation of $\beta$-PP crystals resulting in higher impact strength when compared with the more common a-PP crystals [13]. However, micro-cracks propagate easily in the materials at the impact point due to weak interfacial-bonding between fillers and matrix. Hence, the values of materials IS decreased as result of the presence of these micro-cracks. Thus, inclusion of kenaf-xGNP in the composites formed channels of stress-concentration requiring lower energy to cause cracks within the materials $[4,9]$. Moreover, the stiffening of the polymer chain due to inclusion of kenaf-xGNP in RPP caused reduction in materials ductility and propensity to deform which decreased values of IS with increasing inclusion of kenaf flour. However, the IS behavior of the chemically modified kenaf RPP composites reveals a superior trend when compared with the raw samples implying a better fiber-matrix interfacial adhesion as a result of the chemical modification. Thus, in comparison with the untreated materials, C-TAB modified kenaf flour xGNP-RPP nanocomposites exhibited superior capability to absorb higher energy levels able to hinder propagation of cracks through the materials.

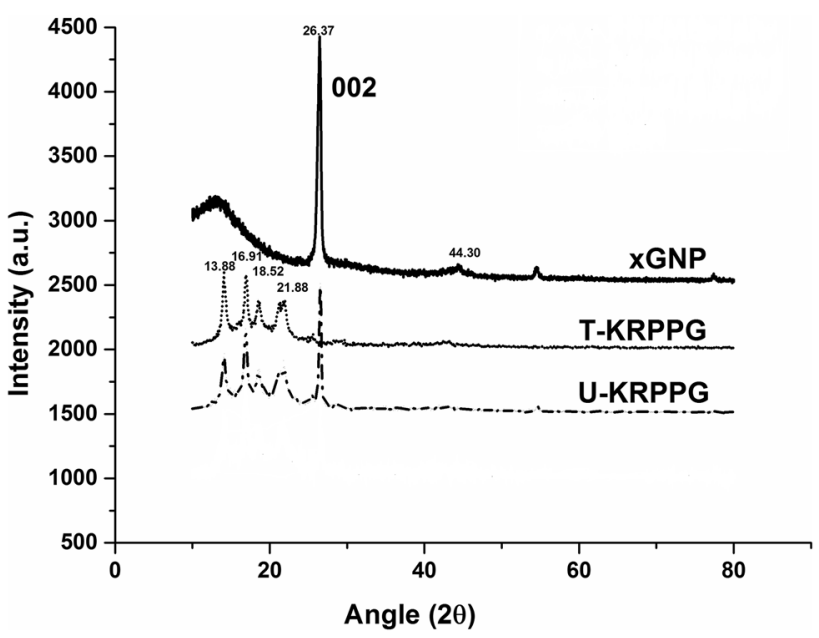

Fig. 9 XRD patterns of XGNP, untreated U-KRPPG and chemically modified T-KRPPG nanocomposites

\subsubsection{X-ray diffraction analysis}

The XRD patterns of XGNP, untreated U-KRPPG and chemically modified T-KRPPG nanocomposites are shown in Fig. 9.

Typically, thermoplastic polymers undergo crystallization to specific crystalline forms. For isotactic PP, there are various packing geometries of PP-helices resulting in four established crystal structures including a-monoclinic, $\beta$-trigonal, $\boldsymbol{\gamma}$-triclinic and $\delta$-smectic crystallographic forms depending on melting chronology, crystallization temperature, presence of extraneous materials and 
cooling rate [13], though the most common crystal is the a-monoclinic crystal form [14]. However, reports have revealed that the $\beta$-form exhibit superior impact strength and toughness due to its unique lamellar morphology, strain induced $\beta$-transition during mechanical deformation, and the formation of large plastic region. As shown Fig. 9 , the peak observed at $2 \theta=16.91^{\circ}$ is the characteristic peak corresponding to (300) plane of $\beta$-form crystals [13]. This peak also occurred due to the inclusion of XGNP in the nanocomposites. A similar observation was reported elsewhere [13]. The second $\beta$-phase peak observed at $2 \theta=21.88^{\circ}$ is for (301) crystallographic plane. Thus, it can be concluded that presence of both graphene nanoplatelets and chemically treated kenaf fibers in RPP effectively enhanced nucleation of $\beta$-crystals which enhanced impact strength and toughness and also enabled secondary reinforcement mechanism. This explains the improvement in impact behavior of the materials at low fibre inclusion and the not very significant drop in impact trend on increasing inclusion of fibre. The inclusion of $x G N P$ induced formation of the extremely narrow peak at $2 \theta=26.37^{\circ}$ (graphene 002 plane) corresponding to interlayer spacing of $0.34 \mathrm{~nm}$. This can be attributed to the homogenous dispersion and exfoliation of $x$ GNP sheets in RPP matrix as revealed in FESEM and TEM images in Figs. 3 and 4 respectively.

\subsubsection{Fourier transform infrared spectroscopy}

FTIR was employed to observe any chemical changes that may occur in the materials. Figure 10 displays the FTIR spectra of U-KRPPG, T-KRPPG and XGNP nanocomposites.

In $x$ GNP spectrum, no visible peaks were observed. The absence of graphite and graphite oxide (GO) bands reveals

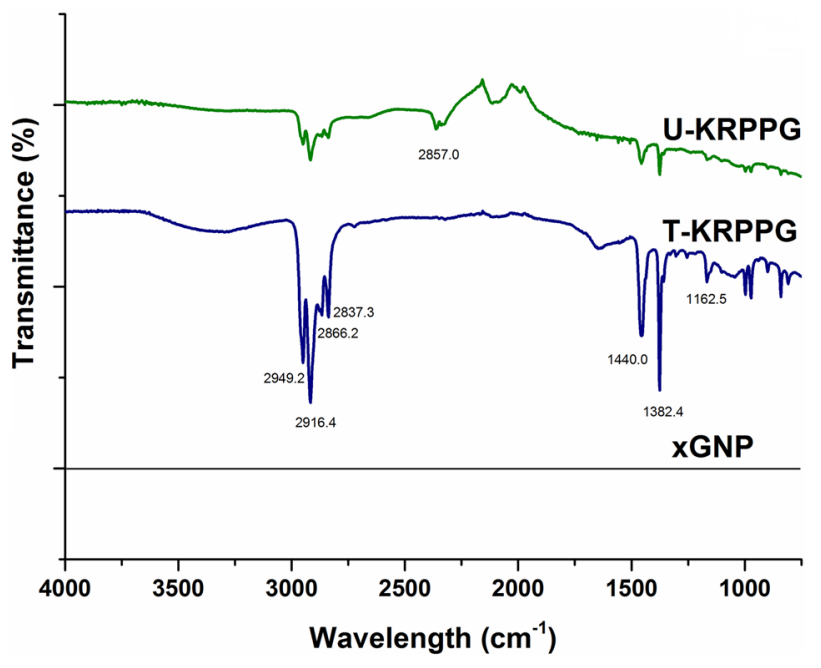

Fig. 10 FTIR spectra of xGNP, U-KRPPG, and T-KRPPG nanocomposites

SN Applied Sciences

SPRINGER NATURE journal the purity of $x G N P$ applied in this work. Thus, the interactions between $x G N P$ and other components are mainly physical because xGNP has nil polar groups. This implies that $x G N P$ is chemically inert and physically interacted in the reaction. Hence, property improvements in the materials are ascribed to physical interactions between xGNPs and other components in addition to chemical modification of treated kenaf nanocomposites which enhanced interfacial adhesion between matrix, xGNP sheets and microfiber. Similar observations were also reported elsewhere $[1,5,15]$. The peak at $1162.5 \mathrm{~cm}^{-1}$ is ascribed to $\mathrm{C}-\mathrm{H}$ outer bending vibration, and in-plane bending and out-of-plane wagging [16], while the peak absorptions at $1382.4 \mathrm{~cm}^{-1}$ is ascribed to the medium $\mathrm{C}-\mathrm{H}$ rock vibrations of alkanes [17]. The absorption peaks at $1440 \mathrm{~cm}^{-1}$ represent the $\mathrm{C}-\mathrm{H}$ bending vibrations of alkenes. The bands at $2837.3,2866.2,2916.4,2949.2 \mathrm{~cm}^{-1}$ are ascribed to the medium intensity of carboxylic $\mathrm{O}-\mathrm{H}$ stretching vibration and the $\mathrm{C}-\mathrm{H}$ stretching of $\mathrm{C}-\mathrm{H}$ bonds [18-23]. From Fig. 10, it is obvious that $\mathrm{OH}$ peak intensity between 3500 and $3100 \mathrm{~cm}^{-1}$ significantly minimized after chemical modification of kenaf flour with $\mathrm{NaOH}$ followed by C-TAB. It is thought that the hydrophilic affinity of kenaf flour significantly minimized, thereby enhancing its interaction with RPP with subsequent improvement of interfacial adhesion. The FESEM and TEM micrographs of the fractured surface of the tensile samples in Figs. $1 \mathrm{~b}$ and $3 \mathrm{~b}$ evidences this phenomenon.

\section{Conclusion}

The effects of chemical modification of kenaf flour in xGNP filled recycled polypropylene hybrid nanocomposites were comparatively studied with the untreated materials. The hybrid nanocomposites were prepared successfully through melt compounding technique using co-rotating twin screw extruder. Morphological and structural studies using FESEM and TEM reveal uniform distribution of xGNP in the matrix. Enhancements in mechanical properties were ascribed to improved interfacial adhesion as a result of kenaf flour modification with $\mathrm{NaOH}$ followed by C-TAB, high graphene platelet modulus (1TPa), efficient stress transfer between matrix, micro-fibrous fillers and $x G N P$, in addition to homogeneous and uniform dispersion of GNP in matrix. From XRD analysis, it may be concluded that presence of both graphene nanoplatelets and kenaf fibers enhanced nucleation of $\beta$-PP crystals which inculcate superior impact strength and toughness and provide secondary reinforcement mechanism. Due to low inclusion of XGNP in nanocomposites, appearance of characteristic xGNP peak implies complete exfoliation and good dispersion of graphene nanosheets in nanocomposites. 
FTIR analysis revealed interactions between xGNP and other components were mainly physical because xGNP has nil polar groups. However, superior improvements in mechanical properties of treated kenaf flour materials were attributed to enhanced interfacial interaction between matrix/kenaf and XGNP sheets. The hybrid xGNP filled RPP kenaf nanocomposites have application potentials in the aerospace, automotive, construction and electronics industries where good strength, stiffness and toughness are essential.

Acknowledgements The authors wish to acknowledge the management of Universiti Teknologi Malaysia for providing enabling infrastructures for the success of this research.

Funding This research received no particular funding. However, the corresponding author received Ph.D. scholarship from Tertiary Education Fund (TETFUND) Nigeria to Universiti Teknologi Malaysia. Authors are grateful to Prof. Charles Esimone, VC Nnamdi Azikiwe University, Awka, Nigeria, and Prof. Azman Hassan of Universiti Teknologi Malaysia.

\section{Compliance with ethical standards}

Conflict of interest The authors hereby declare no conflict of interests.

Ethical approval This research was conducted on materials with strict adherence to safe laboratory ethics, policies and practices.

\section{References}

1. Chaudhary V, Bajpai P, Maheshwari S (2018) Studies on mechanical and morphological characterization of developed jute/ hemp/flax reinforced hybrid composites for structural applications. J Nat Fibers 15:80-97. https://doi.org/10.1080/15440 478.2017.1320260

2. Mohamed S, Zainudin E, Sapuan S, Azaman M, Arifin A (2018) In: Sapuan SM, Ismail H, Zainudin ES (eds) Natural fibre reinforced vinyl ester and vinyl polymer composites. Woodhead Publishing Series in Composites Science and Engineering, Woodhead Publishing, pp 1-25. https://doi.org/10.1016/B978-0-08-10216 $0-6.00001-9$

3. Le Moigne N, Otazaghine B, Corn S, Angellier-Coussy H, Bergeret A (2018) Surfaces and interfaces in natural fibre reinforced composites. Springer Briefs in Molecular Science, Springer, Cham

4. Wu Y, Xia C, Cai L, Garcia A, Shi S (2018) Development of natural fiber-reinforced composite with comparable mechanical properties and reduced energy consumption and environmental impacts for replacing automotive glass-fiber sheet molding compound. J Clean Prod 184:92-100

5. Bhattacharjee S, Bajwa D (2018) Degradation in the mechanical and thermo-mechanical properties of natural fiber filled polymer composites due to recycling. Constr Build Mater 172:1-9. https://doi.org/10.1016/j.conbuildmat.2018.03.010

6. Sanjay M, Madhu P, Jawaid M, Senthamaraikannan P, Senthil S, Pradeep S (2018) Characterization and properties of natural fiber polymer composites: A comprehensive review. J Clean Prod 172:566-581

7. Rajini N, Winowlin J, Siva I, Rajulu A, Rajakarunakaran S (2016) Fire and thermal resistance properties of chemically treated ligno-cellulosic coconut fabric-reinforced polymer eco-nanocomposites. J Ind Text 47(1):104-124

8. Lau K, Hung P, Zhu M, Hui D (2018) Properties of natural fibre composites for structural engineering applications. Composites Part B: Engineering 136:222-233

9. Michael M, El-Zaher N, Ibrahim S (2004) Investigation into surface modification of some polymeric fabrics by UV/ozone treatment. Polym-Plast Technol Eng 43(4):1041-1052. https:// doi.org/10.1081/PPT-200030016

10. Idumah C, Hassan A (2017) Hibiscus cannabinus fiber/PP based nano-biocomposites reinforced with graphene nanoplatelets. J Nat Fibers 14(5):691-706

11. Sreenivasan S, Sulaiman S, Ariffin M, Baharudin B, Abdan K (2018) Physical properties of novel kenaf short fiber reinforced bulk molding compounds (BMC) for compression moulding. Mater Today Proc 5(1):1226-1232

12. Idumah C, Hassan A (2016) Emerging trends in eco-compliant, synergistic, and hybrid assembling of multifunctional polymeric bionanocomposites. Rev Chem Eng 32(3):305-361

13. Savetlana S, Mulvaney-Johnson L, Gough T, Kelly A (2018) Plastics. Rubber Compos 47:77-86. https://doi.org/10.1080/14658 011.2017.1418711

14. Brunauer S, Emmett PH, Teller E (1938) J Am Chem Soc 60(2):309-319

15. Vaisanen T, Batello P, Lappalainen R, Tomppo L (2018) Modification of hemp fibers (Cannabis sativa L.) for composite applications. Ind Crops Prod 111:422-429

16. Idumah C, Hassan A. Emerging trend in flame retardancy of biofibres, biopolymers, biocomposites and bionanocomposites. Rev Chem Eng. https://doi.org/10.1515/revce-2015-0017

17. Idumah C, Hassan A (2018) Effect of exfoliated graphite nanoplatelets on thermal and heat deflection properties of kenaf polypropylene hybrid nanocomposites. J Polym Eng 36(9):877-889

18. Yallew T, Aregawi S, Kumar P, Singh I (2018) Response of natural fiber reinforced polymer composites when subjected to various environments. Int J Plast Technol 22(1):56-72

19. Suvari F, Ulcay Y, Maze B (2013) Acoustical absorptive properties of spunbonded nonwovens made from islands-in-the-sea bicomponent filaments. J Text Inst 104:438-445

20. Sepe R, Bollino F, Boccarusso L, Caputo F (2018) Influence of chemical treatments on mechanical properties of hemp fiber reinforced composites. Compos B Eng 133:210-217

21. Sinha A, Narang H, Bhattacharya $S$ (2018) Evaluation of bending strength of abaca reinforced polymer composites. Mater Today Proc 5(2):7284-7288

22. Bar M, Alagirusamy R, Das A (2018) In: Fangueiro R, Rana S (eds) Advances in natural fibre composites. Springer, Cham

23. Idumah C, Hassan A, Bourbigot S (2017) Influence of exfoliated graphene nanoplatelets on flame retardancy of kenaf flour polypropylene hybrid nanocomposites. J Anal Appl Pyrol 123:65-72

Publisher's Note Springer Nature remains neutral with regard to jurisdictional claims in published maps and institutional affiliations. 